\title{
A Format-preserving encryption FF1, FF3-1 Using Lightweight Block Ciphers LEA and, SPECK
}

\author{
Wonyoung Jang \\ Dept. of Computer Science\& \\ Engineering \\ Soonchunhyang University \\ Asan, South Korea \\ ozragwort@sch.ac.kr
}

\author{
Sun-Young Lee \\ Dept. of Information Security \\ Engineering \\ Soonchunhyang University \\ Asan, South Korea \\ sunlee@sch.ac.kr
}

\begin{abstract}
Format-preserving encryption has been studied for a long
ABSTRACT
Format-preserving encryption has been studied for a long time since its proposal, but the algorithm is yet to be adequately evaluated or verified. The existing standard format-preserving encryption FF1 and FF3-1 use block cipher AES in the internal function. This paper proposes a method to improve the speed of FF1 and FF3-1 whereby the algorithm is implemented by changing the cipher to lightweight block ciphers LEA(Lightweight Encryption Algorithm) and SPECK. The encryption speed is analyzed and compared with that of the existing encryption by dividing it into high-performance computer environments and low-performance Internet of Things environments. The results showed that the encryption speed was improved compared with FF1 and FF3-1. Improving the encryption speed of format-preserving encryption will make it easier to apply format-preserving encryption to various systems.
\end{abstract}

\section{CCS CONCEPTS}

- Security and privacy $\rightarrow$ Block and stream ciphers

\section{KEYWORDS}

Format-preserving encryption, FF1, FF3-1, LEA, SPECK, Lightweight Block Cipher
Permission to make digital or hard copies of part or all of this work for personal or classroom use is granted without fee provided that copies are not made or distributed for profit or commercial advantage and that copies bear this notice and the full citation on the first page. Copyrights for third-party components of this work must be honored. For all other uses, contact the owner/author(s). To copy otherwise, or republish, to post on servers or to redistribute to lists, requires prior specific permission and/or a fee.

SAC 20, March 30 -April 3, 2020, Brno, Czech Republic

(C) 2020 Copyright held by the owner/author(s). 978-1-4503-6866-7/20/03. . $\$ 15.00$

DOI: $10.1145 / 3341105.3373953$ .

西

(1)

Format-preserving encryption(FPE) is an encryption algorithm that maintains ciphertext length and text form. As FPE does not change the length and form, it is suitable for encrypting data with a finite length or format. FPE was first proposed by Michael Brightwell et al [1]. Subsequently, three types of prefix cipher, cycle-walking cipher, and generalizedFeistel cipher were presented, and a method using an Rankthen-Encipher(RTE) and an unbalanced Feistel structure was proposed [2-3]. RTE is a method that enables FPE to compatible to various formats [3]. Since then, the algorithms of FFX, VAES3, and BPS have been proposed as standards FF1, FF2, and FF3 [4-7]. However, the vulnerability of FF2 was discovered and excluded, and FF3 was modified to follow the FF1 standard with the new version being named FF3-1 [8-10]. Although format-preserving encryption has been studied for a long time, the study of the performance and the new algorithm is insufficient. In this paper, we proposed a method to improve the encryption speed of NIST standard format-preserving encryption FF1 and FF3-1 using lightweight block ciphers, and compared and analyzed the performance. Instead of the AES algorithms of FF1 and FF3-1, the lightweight block cipher algorithm LEA and SPECK are used. LEA is an algorithm that encrypts 128-bit data blocks and consists of only 32-bit unit ARX (Addition, Rotation, XOR) operations, which operate at high speed on a general-purpose 32-bit software platform that supports these operations. In addition, the ARX operation arrangement in the round function ensures safety and eliminates the use of S-box at the same time, enabling lightweight implementation [11]. SPECK has the Feistel structure and ARX form. There is a block size of 32/48/64/128 bits and a key size of $64 / 72,96 / 96,128 / 96,144 / 128,192,256$, respectively. SPECK has many block size and key sizes to be applied, so it can be used in various environments and has an ARX structure to enable highspeed operation [12]. If FF1 and FF3-1 algorithms are 
implemented using lightweight encryption that is faster than AES, encryption speed is faster than FF1 and FF3-1 algorithms using AES.

In Section 2, we describe the FF1 and FF3-1 algorithms. In Section 3 , we explain how to apply a lightweight block cipher and compare and analyze the speed of the proposed method in highperformance computer environments and loT environments. Finally, in Section 4, we conclude the paper.

\section{Format-preserving encryption FF1 and FF3-1}

\subsection{Feistel structure of FF1 and FF3-1}

The internal functions of FF1 and FF3 are based on AES-128, and both algorithms have the Feistel structure. Fig. 1 represents the Feistel structure of FF1 and FF3-1.

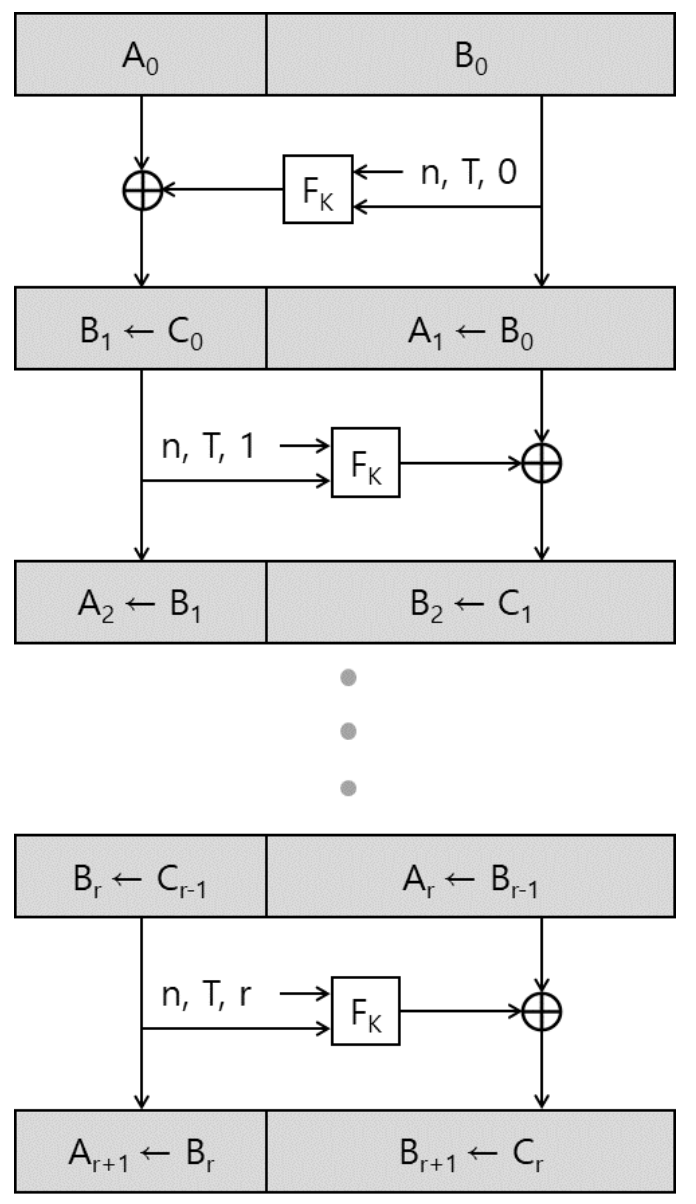

Figure 1: Feistel structure of FF1 and FF3-1

In Fig. $1, \mathrm{n}$ is the length of the entire message, $\mathrm{r}$ is the round number, and $T$ is Tweak. To use the NIST standard formatpreserving encryption algorithm, plain text, 128bit AES key, and Tweak are required. First, the data is split into two parts, then one part is computed with $\mathrm{n}$, Tweak, and round numbers in the round function $\left(F_{K}\right)$; and swapped with the other. This process repeats for $r$ rounds where FF1 is the 10th round FF3-1 is the 8th round [10].

\subsection{Field definition}

The definition of the fields used in the subsequent Algorithm formula is as shown in Table 1.

Table 1: Field definition

\begin{tabular}{|c|c|}
\hline FIELD & DESCRIPTION \\
\hline radix & $\begin{array}{l}\text { Number of elements in domains used in } \\
\text { plaintext }\end{array}$ \\
\hline minlen & Minimum length of message \\
\hline maxlen & Maximum length of message \\
\hline maxTlen & Maximum length of Tweak \\
\hline $\mathrm{CIPH}_{\mathrm{K}}(\mathrm{X})$ & Encrypt X using key $\mathrm{K}$ \\
\hline $\operatorname{NUM}_{\text {radix }}(\mathrm{X})$ & $\begin{array}{l}\text { transforming the radix- notation } \\
\text { number } \mathrm{X} \text { into a decimal integer }\end{array}$ \\
\hline $\operatorname{NUM}(X)$ & $\begin{array}{l}\text { transforming the bit string } \mathrm{X} \text { into a } \\
\text { decimal number }\end{array}$ \\
\hline $\mathrm{STR}_{\text {radix }}(\mathrm{X})$ & $\begin{array}{l}\text { transforming the decimal } \mathrm{X} \text { into a } \\
\text { number of radix and } \mathrm{m} \text { in length }\end{array}$ \\
\hline $\operatorname{REV}(\mathrm{X})$ & $\begin{array}{l}\text { transforming the numeric string } \mathrm{X} \text { into } \\
\text { inverse order }\end{array}$ \\
\hline $\operatorname{PRF}(\mathrm{X})$ & $\begin{array}{l}\text { Use } \mathrm{X} \text { as the initial block of } \mathrm{CBC} \\
\text { encryption mode }\end{array}$ \\
\hline
\end{tabular}

\section{$2.3 \quad$ FF1}

FF1 is a format-preserving encryption derived from the FFX mode. Table 2 shows the requirements of FF1.

Table 2: Requirements of FF1

\begin{tabular}{|l|l|}
\hline radix & {$\left[2 \ldots 2^{16}\right]$} \\
\hline radix & more than $1,000,000$ \\
\hline minlen & 2 \\
\hline maxlen & $2^{32}$ \\
\hline round & 10 \\
\hline
\end{tabular}


The FFX mode limits the message length to less than 128 bits, but FF1 can have a minimum length of 2 , a maximum length of 232 , and a Tweak length of $2^{32}$. The range of the radix is between 2 and $2^{16}$ or less, however radix minlen should exceed $1,000,000$. In the FFX mode, the number of rounds recommended by the length of the message was determined, but FF1 was fixed to 10 rounds[4,7]. Algorithm 1 shows the encryption process of FF1; when you receive plain text $\mathrm{X}$, you split it into A and B. b (the length of B in the internal function), $\mathrm{d}$, and $\mathrm{P}$ (the initial block) used for the after encryption are established. Inside of the round function $\mathrm{T}$ means the tweak value and i represents the round number. $Q$ is produced by using T, $\mathrm{i}$, and B. By using the initial blocks $\mathrm{P}$ and $\mathrm{Q} R$ is created and is encrypted and $\mathrm{S}$ is created. $\mathrm{S}$ and $\mathrm{A}$ are converted into the decimal string and it adds on the mod radix $^{m}$. Then, the step of converting the original m-length radix frequency value is repeated 10 rounds and the value connecting the last $\mathrm{A}$ and $\mathrm{B}$ values becomes the encryption value. Algorithm 2 shows the decryption process of FF1 and is the reverse of encryption [10].
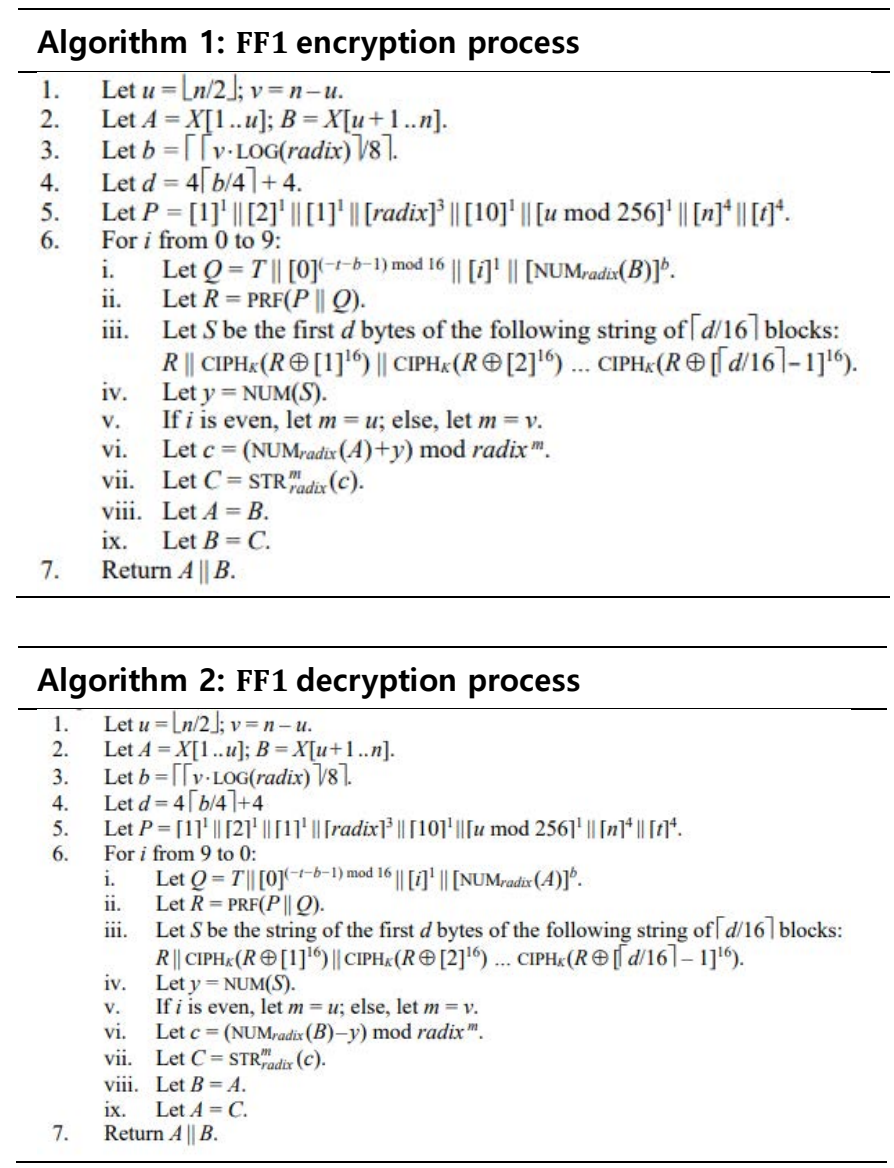

\section{$2.4 \quad$ FF3-1}

FF3-1 is format-preserving encryption derived from BPS and has a structure similar to CBC mode in block encryption mode. The BPS was designed to use standardized algorithms such as TDEA, AES, and SHA-2, but was modified to use AES as it was standardized as FF3[6-7,10]. Table 3 shows the requirements of FF3-1.

Table 3: Requirements of FF3-1

\begin{tabular}{|l|l|}
\cline { 2 - 2 } radix & {$\left[2 \ldots 2^{16}\right]$} \\
\hline $\begin{array}{l}\text { radix } \\
\text { minlen } \\
\text { maxlen }\end{array}$ & more than $1,000,000$ \\
\cline { 2 - 2 } & 2 \\
\cline { 2 - 2 } round & $2 \times\left\lfloor\log _{\text {radix }}\left(2^{96}\right)\right\rfloor$ \\
\hline
\end{tabular}

The radix and the minimum length are the same as FF1. The maximum length is $2 \times\left[\log _{\text {radix }}\left(2^{96}\right)\right]$. FF3-1 has an 8-round Feistel structure unlike FF1. Algorithm 3 shows the encryption process of FF3-1. The algorithm divides plain text $\mathrm{X}$ into $\mathrm{A}$ and $\mathrm{B}$, in the same way as FF1.

The Tweak value is fixed at $54 \mathrm{bit}, \mathrm{T}_{\mathrm{L}}$ is set to $\mathrm{T}[0 . .27] \| 0^{4}$, and $\mathrm{T}_{\mathrm{R}}$ is set to $\mathrm{T}[32 . .55]\|\mathrm{T}[28 . .31]\| 0^{4}$. The number of rounds in the round function is set to $i$, and if $i$ is even, $m=u, W=T_{R}$, and if it is odd, $m=v, W=T_{L}$. A four-byte value is made using $W$ and $i$. After changing the radix-notation number $B$ to the reverse order, fill the front with 0 until there are 12 bytes of data and connect two to generate P. By using $\mathrm{S}$ and $\mathrm{A}$ in the same way as $\mathrm{FF} 1, \mathrm{C}$ is created. The value is found by repeating the round function for 8 rounds. The cipher text is the value of the connection to the final $\mathrm{A}$ and $\mathrm{B}$. Algorithm 4 shows the decryption process of FF3-1, which is the reverse of encryption [10].

\footnotetext{
Algorithm 3: FF3-1 encryption process

1. Let $u=\lceil n / 2\rceil ; v=n-u$.

2. Let $A=X\lceil 1 \ldots u\rceil: B=X\lceil u+1 . . n\rceil$.

3. Let $T_{L}=T[0 . .27] \| 0^{4}$ and $T_{R}=T[32 . .55]\|T[28 . .31]\| 0^{4}$.

4. For $i$ from 0 to 7 :

i. If $i$ is even, let $m=u$ and $W=T_{R}$, else let $m=v$ and $W=T_{L}$.

ii. Let $P=W \oplus[i]^{4} \|\left[\operatorname{NUM}_{\text {radix }}(\operatorname{REV}(B))\right]^{12}$.

iii Let $S=\operatorname{REVB}\left(\operatorname{CIPH}_{\mathrm{REVB}(K)} \operatorname{REVB}(P)\right)$.

iv. Let $y=\operatorname{NUM}(S)$.

v. Let $c=\left(\operatorname{NUM}_{\text {radix }}(\operatorname{REV}(A))+y\right) \bmod$ radix $^{m}$.

vi. Let $C=\operatorname{REV}\left(\operatorname{STR}_{\text {radix }}^{m}(c)\right)$.

vii. Let $A=B$.

viii. Let $B=C$.

5. Return $A \| B$.
} 


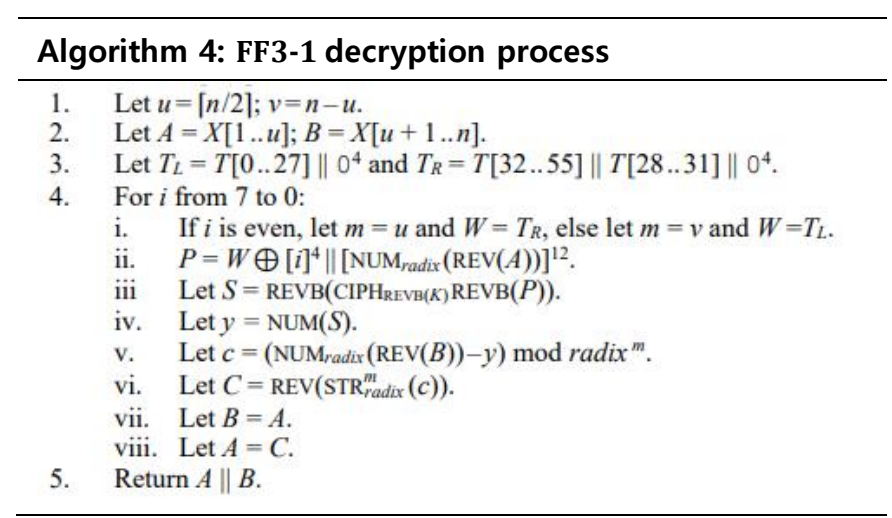

\section{PROPOSED METHOD}

\subsection{Format-preserving encryption applied with lightweight block cipher}

In this paper, we propose an algorithm that changes AES, which is used in the $\mathrm{CIPHK}_{\mathrm{K}}(\mathrm{X})$ phase of FF1 and FF3-1, to LEA and SPECK, which are lightweight block ciphers. We modified the $\mathrm{CIPH}_{\mathrm{K}}(\mathrm{X})$ of Algorithm 1, Algorithm 2, 6. iii and Algorithm 3 , Algorithm 4, 4. iii. The existing $\mathrm{CIPH}_{\mathrm{K}}(\mathrm{X})$ of FF1 and FF3-1 is a step of encrypting using AES and modified CIPHк(X) of FF1 and FF3-1 is a step of encrypting using LEA and SPECK, which are lightweight block ciphers. LEA and SPECK are defined as FF1-LEA, FF1-SPECK, FF3-1-LEA, FF3-1-SPECK, respectively.

\subsection{Experimental environment}

To test the encryption speed of the proposed method, we tested it in a high-performance computer (I7-8700) environment and an IoT (ARM Cortex A7) environment. Table 4 lists the specifications of the high-performance computer, and Table 5 lists those of the IoT environment.

Table 4: Experimental (High-performance)

\begin{tabular}{cc}
\hline CPU & $17-8700(3.20 \mathrm{GHz})$ \\
Core/Thread & $6 / 12$ \\
Memory & $16.0 \mathrm{~GB}$ \\
OS & Windows10 (64bit) \\
\hline
\end{tabular}

Table 5: Experimental (IoT)

$\begin{array}{cc}\text { CPU } & \text { ARM Cortex-A7 } \\ (0.9 \mathrm{GHz})\end{array}$

\begin{tabular}{cc} 
Core & $1-4$ \\
Memory & $1.0 \mathrm{~GB}$ \\
OS & Raspbian 10 \\
\hline
\end{tabular}

\subsection{Speed comparison of the proposed method for FF1}

Fig. 2 shows the encryption speed comparison of the proposed method concerning FF1 in a high-performance computer environment; Fig. 3 shows the encryption speed comparison of the proposed method concerning FF1 in an IoT environment.

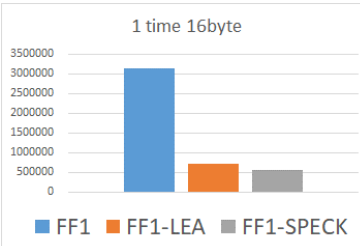

(a) 16byte - 1 time

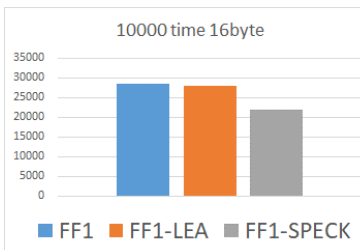

(c) 16byte - 10,000 times

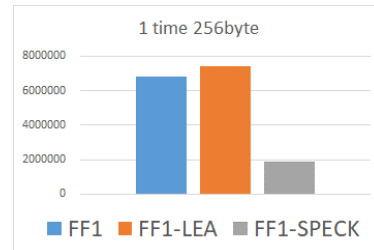

(b) 256byte - 1 time

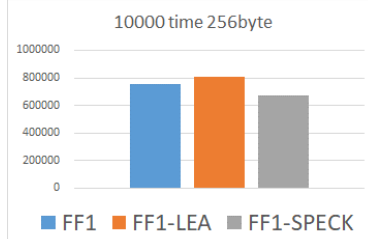

(d) 256byte - 10,000 times
Figure 2: Encryption Time of FF1, FF1-LEA, FF1-SPECK in a High-Performance Computer Environment

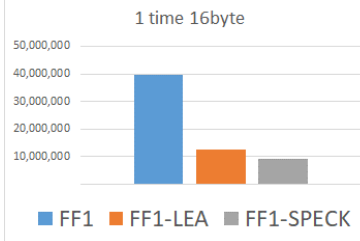

(a) 16byte - 1 time

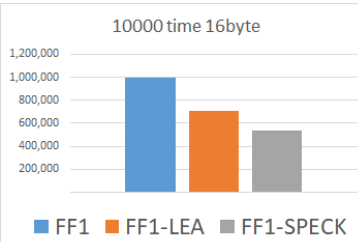

(c) 16byte - 10,000 times

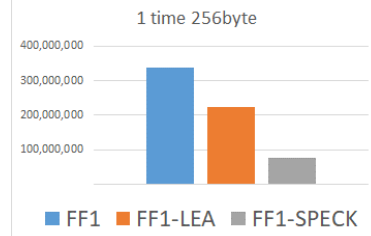

(b) 256byte - 1 time 10000 time 256byte

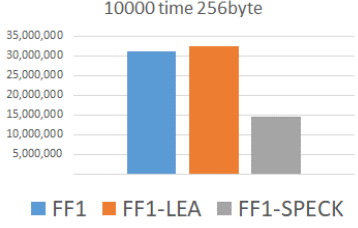

(d) 256byte - 10,000 times
Figure 3: Encryption Time of FF1, FF1-LEA, FF1-SPECK in an IoT Environment 
When testing in a high-performance computer environment, Fig. 2-(a) shows that the encryption time is reduced by applying LEA and SPECK to FF1. However, Fig. 2-(c) shows that the efficiency of using lightweight block ciphers decreases when encryption is repeated 10,000 times. Fig. 2-(b) and Fig. 2-(d) shows that the longer the text, the slower FF1-LEA may be. Fig. 3 shows that the proposed method improves speed in IoT environment. Fig. 3 shows the faster encryption speed in the IoT environment except for FF1-LEA in Fig. 3-(d). Experimental results show that the FF1 algorithm greatly improves the encryption speed when applying LEA and SPECK when encrypting short data. However, if the length of the plain text is lengthened or it encrypts several times. the rate of improvement of the encryption speed is lowered.

\subsection{Speed comparison of the proposed method for FF3-1}

Fig. 8 shows the encryption speed comparison of the proposed method concerning FF3-1 in a high performance computer environment; Fig. 9 shows the encryption speed comparison of the proposed method concerning FF3-1 in an IoT environment.

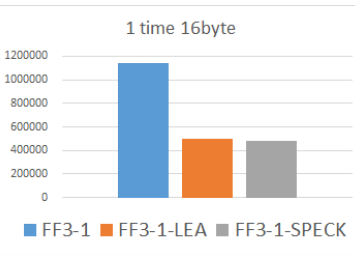

(a) 16byte - 1 time

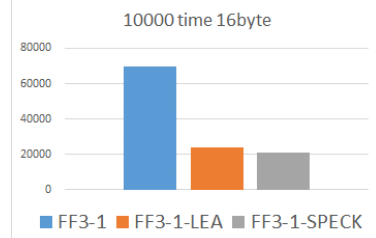

(c) 16byte - 10,000 times

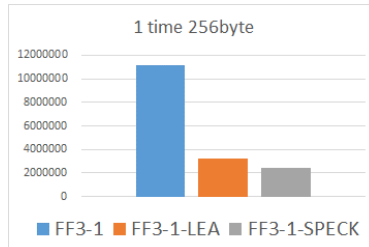

(b) 256byte - 1 time

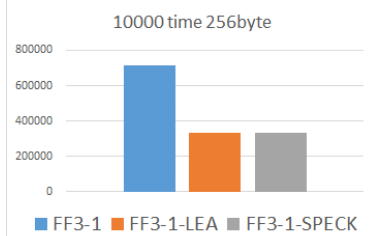

(d) 256byte - 10,000 times
Figure 4: Encryption Time of FF3-1, FF3-1-LEA, FF3-1SPECK in a High-Performance Computer Environment

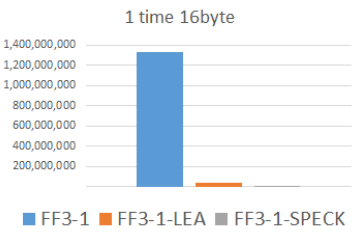

(a) 16byte - 1 time

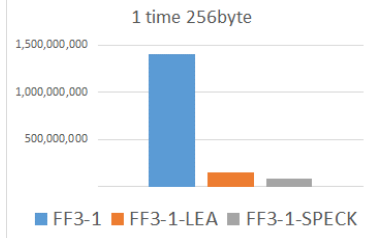

(b) 256byte - 1 time

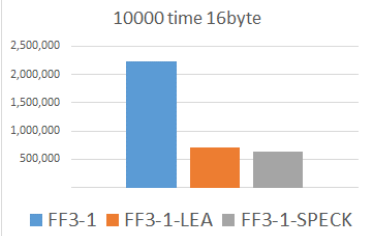

(c) 16byte - 10,000 times

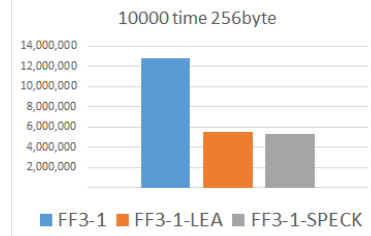

(d) 256byte - 10,000 times
Figure 5: Encryption Time of FF3-1, FF3-1-LEA, FF3-1SPECK in an IoT Environment

Fig. 4 and Fig. 5 show that when the proposed method is applied, the encryption speed is greatly improved in all situations. Unlike FF1, the encryption speed is improved even if the length of the plain text is lengthened or the encryption occurs several times.

\subsection{Experiment result}

Table 6 and Table 7 are tables that summarize the encryption speeds of FF1, FF3-1, and proposed methods in highperformance computer environments and IoT environments. Table 7 and Table 9 are tables that show the rate of encryption speed improvement of the proposed method based on Table 6 and Table 8.

Table 6: Encryption Time by algorithm in high performance computer environment

\begin{tabular}{ccccc}
\hline \hline (ns) & \multicolumn{2}{c}{10000} & & 1 \\
& 16byte & 256 byte & 16 byte & 256 byte \\
\hline FF1 & 28627 & 757160 & 3149000 & 6833000 \\
FF1-LEA & 28093 & 811092 & 710600 & 7422300 \\
FF1-SPECK & 21863 & 670681 & 564000 & 1901500 \\
\hline FF3-1 & 69451 & 712434 & 1142700 & 11132600 \\
FF3-1-LEA & 24034 & 330584 & 499800 & 3182700 \\
FF3-1-SPECK & 20743 & 330531 & 483700 & 2386900 \\
\hline \hline
\end{tabular}

Table 7: Speed improvement rate by algorithm in high performance computer environment

\begin{tabular}{ccccc}
\hline \hline \multirow{2}{*}{$\%)$} & \multicolumn{2}{c}{10000} & \multicolumn{2}{c}{1} \\
& 16byte & 256byte & 16byte & 256byte \\
\hline FF1-LEA & 1.9 & -6.6 & 343.1 & -7.9
\end{tabular}




\begin{tabular}{ccccc} 
FF1-SPECK & 30.9 & 12.9 & 458.3 & 259.3 \\
FF3-1-LEA & 189.0 & 115.5 & 128.6 & 249.8 \\
FF3-1-SPECK & 234.8 & 115.5 & 136.2 & 366.4 \\
\hline \hline
\end{tabular}

Table 8: Encryption Time by algorithm in IoT environment

\begin{tabular}{|c|c|c|c|c|}
\hline \multirow{2}{*}{ (ns) } & \multicolumn{2}{|c|}{10000} & \multicolumn{2}{|c|}{1} \\
\hline & 16byte & 256byte & 16byte & 256byte \\
\hline FF1 & 999,940 & $\begin{array}{c}31,045,67 \\
3\end{array}$ & $\begin{array}{c}39,805,88 \\
4\end{array}$ & $\begin{array}{c}339,111,5 \\
53\end{array}$ \\
\hline FF1-LEA & 710,618 & $\begin{array}{c}32,414,89 \\
9\end{array}$ & $\begin{array}{c}12,412,29 \\
1\end{array}$ & $\begin{array}{c}222,983,7 \\
96\end{array}$ \\
\hline FF1-SPECK & 531,549 & $\begin{array}{c}14,469,77 \\
2\end{array}$ & $9,085,469$ & $\begin{array}{c}77,172,34 \\
1\end{array}$ \\
\hline FF3-1 & $2,225,679$ & $\begin{array}{c}12,789,10 \\
3\end{array}$ & $\begin{array}{c}1,331,038 \\
713\end{array}$ & $\begin{array}{c}1,400,889 \\
597\end{array}$ \\
\hline FF3-1-LEA & 706,018 & $5,505,764$ & $\begin{array}{c}31,551,56 \\
2\end{array}$ & $\begin{array}{c}144,927,2 \\
35\end{array}$ \\
\hline FF3-1-SPECK & 625,779 & $5,345,052$ & $8,243,542$ & $\begin{array}{c}82,173,90 \\
4\end{array}$ \\
\hline
\end{tabular}

Table 9: Speed improvement rate by algorithm in IoT environment

\begin{tabular}{ccccc}
\hline \hline \multirow{2}{*}{$\%)$} & \multicolumn{2}{c}{10000} & \multicolumn{2}{c}{1} \\
& 16byte & 256byte & 16byte & 256byte \\
\hline FF1-LEA & 40.7 & -4.2 & 220.7 & 52.1 \\
FF1-SPECK & 88.1 & 114.6 & 338.1 & 339.4 \\
FF3-1-LEA & 215.2 & 132.3 & 4118.6 & 866.6 \\
FF3-1-SPECK & 255.7 & 139.3 & 16046.4 & 4604.8 \\
\hline \hline
\end{tabular}

Table 7 shows FF3-1-LEA with a lightweight block cipher improving the speed by a minimum of $115.5 \%$ and a maximum of $249.8 \%$ in high performance computer environment. FF1 has improved speed when encrypting data once, but has been slowed down when encryption data is large or encryption is done several times. Table 9 shows that the proposed encryption method has a higher encryption rate in an IoT environment than in a high-performance computer environment. When the proposed method is applied, both environments show that FF3-1 has better encryption speed than FF1. In other words, the proposed method of formatpreserving encryption applied lightweight block cipher is more efficient than FF1 when applied to FF3-1. In addition, the proposed method is considered to be more efficient in a lower-performance environment than in a high-performance computer environment.

\section{CONCLUSION}

In this study, we proposed a method to improve encryption speed by applying a lightweight block cipher to FF1 and FF3-1 format-preserving encryption. Four new algorithms were proposed: FF1-LEA, FF1-SPECK, FF3-1-LEA, and FF3-1-SPECK by changing AES, which is used in the $\mathrm{CIPH}_{\mathrm{K}}(\mathrm{X})$ phase of $\mathrm{FF} 1$ and FF3-1, to the lightweight block ciphers LEA and SPECK. The test was conducted in a high-performance computer environment and a low-performance IoT environment, and the encryption speed was compared and analyzed with that of the existing FF1 and FF3-1 by changing the message size and the number of encryption rounds. Experimental results show that the proposed method improves the encryption speed significantly compared to the existing FF1 and FF3-1. The encryption is also more efficient in low-performance IoT environments than high-performance computer environments, and more efficient when applied to FF3-1 than FF1. Like the experiment result, if encryption speed of formatpreserving encryption is improved, we expect that formatpreserving encryption can be used efficiently in various systems.

\section{ACKNOWLEDGMENTS}

This work was supported by the National Research Foundation of Korea (NRF) grant funded by the Korea government (MSIT) (No. 2018R1A4A1025632) and by the Basic Science Research Program through the National Research Foundation of Korea (NRF) that is funded by the Ministry of Education (NRF-2018R1D1A1B07047656).

\section{REFERENCES}

[1] Michael Brightwell, and H.Smith. 1997. Using Datatype Preserving Encryption To Enhance Data Warehouse Security. 20th National Information Systems Security Conference Proceeding(NISSC). 141-149.

[2] John Black, Phillip Rogaway. 2002. Ciphers with Arbitrary Finite Domains. In Cryptographers 'Track at the RSA Conference. Springer, Berlin, Heidelberg 114-130.

[3] Mihir Bellare, Thomas Ristenpart, Phillip Rogaway, and Till Stegers. 2009. Format-preserving encryption. International Workshop on Selected Areas in Cryptography. Springer, Berlin, Heidelberg. 295-312.

[4] Bellare, Mihir, Phillip Rogaway, and Terence Spies. 2010. The FFX mode of operation for Format-preserving encryption. NIST submission, 20.

[5] Joachim Vance. 2011. VAES3 scheme for FFX: An addendum to The FFX mode of operation for Format Preserving Encryption. NIST submission, 6, 7.

[6] Brier, Eric, Thomas Peyrin, and Jacques Stern. 2010. BPS: a Format-preserving encryption proposal. Режим доступу до ресурсу. DOI: http://csrc.nist.gov/groups/ST/toolkit/BCM/documents/proposedmodes/bp s/bps-spec.pdf

[7] Dworkin, Morris. 2016. Recommendation for block cipher modes of operation: methods for formatpreserving encryption. NIST Special Publication 800:38G. DOI: https://doi.org/10.6028/NIST.SP.800-38G

[8] Dworkin, Morris J, and Ray A. Perlner. 2015. Analysis of VAES3 (FF2). No. 
Cryptology ePrint Archive.

[9] F. B. Durak, S. Vaudenay. 2017. Breaking the FF3 Format-preserving encryption Standard Over Small Domains. Advances in Cryptology-CRYPTO 2017. Lecture Notes in Computer Science vol. 10402. Springer. 679-707. DOI: https://doi.org/10.1007/978-3-319-63715-0_23

[10] Dworkin, Morris. 2019. Recommendation for Block Cipher Modes of Operation: Methods for Format-preserving encryption. NIST Special Publication 800:38G Rev. 1. DOI: https://doi.org/10.6028/NIST.SP.800-38Gr1draft

[11] Deukjo Hong, Jung-Keun Lee, Dong-Chan Kim, Daesung Kwon, Kwon Ho Ryu, and Dong-Geon Lee. 2013. LEA: A 128-Bit Block Cipher for Fast Encryption on Common Processors. International Workshop on Information Security Applications. Springer, Cham. 3-27.

[12] Ray Beaulieu, Stefan Treatman-Clark, Douglas Shors, Bryan Weeks, Jason Smith, and Louis Wingers. 2015. The SIMON and SPECK lightweight block ciphers. 2015 52nd ACM/EDAC/IEEE Design Automation Conference (DAC). 1-6. DOI: https://doi.org/10.1145/2744769.2747946 